\title{
Investigations of a new Free Piston Expander Engine Cycle
}

\author{
B. S. Preetham ${ }^{\mathrm{a}}$, L. Weiss ${ }^{\mathrm{b}, *}$ \\ ${ }^{a}$ Department of Aerospace and Mechanical Engineering, University of Notre Dame, Notre \\ Dame, IN 46556 USA \\ ${ }^{b}$ Institute for Micromanufacturing, Department of Mechanical Engineering, Louisiana Tech \\ University, Ruston, LA 71272 USA
}

\begin{abstract}
This work examines the design and operation of a new, small-scale Free Piston Expander (FPE) engine that operates using low temperature waste heat sources to produce useful power output. The FPE is based on a sliding-piston architecture that eliminates challenges associated with MEMS-based rotating systems. A nonlinear lumped-parameter model is derived to study the factors that control the performance of the FPE engine and its unique operating cycle. This basic analysis considers a closed cycle operation of the FPE with low thermal or heat inputs and dimensions on the order of several millimeters. Key system design and operating parameters such as piston mass, external load, and heat input are varied to identify conditions and trends for optimal performance. The model indicated the pressure-volume diagram resembles a constant pressure cycle for a certain set of operating conditions but is also condition dependent. Increased heat inputs to the FPE reduced the engine natural or operating frequency while increasing the power output. Thermal efficiencies of the FPE are shown to be predictably low, on the order of $0.2 \%$ due to the small heat input and operating temperature gradients associated with waste heat. Key design features are identified that reveal FPE efficiency, operating frequency, and output power are dependent on piston mass, external load, input heat-rate, and duration of heat input.
\end{abstract}

Keywords: MEMS, reciprocating engine, thermal scavenge, steam system, volumetric expander

\section{Introduction}

There are many significant challenges that influence energy supply, policy, and future consumption trends. At the heart of these challenges is increased

\footnotetext{
*Corresponding author

Email address: lweiss@latech.edu (L. Weiss)
}

Preprint submitted to Energy

February 25, 2016

(C) 2016. This manuscript version is made available under the Elsevier user license http://www.elsevier.com/open-access/userlicense/1.0/ 
worldwide energy consumption. The U.S. Energy Information Agency (EIA)

5 has predicted that world consumption will increase $56 \%$ by 2050 compared to 2010 levels [1]. In the midst of the predicted energy consumption growth exists a competing need to reduce greenhouse gas (GHG) emissions [2]. There is acknowledgement that renewable energy supplies will provide a growing contribution to worldwide energy needs, however, traditional non-renewables will remain a significant source for the foreseeable future [1. This indicates a continued need for traditional cycle efficiency and thermal management improvement 3.

Thermodynamic cycles operating from non-renewable sources include the standard Otto cycle with average efficiency of about 35\% 4]. The majority of energy is lost to the surrounding environment as low temperature waste heat via heat rejection. This reality is similar for other industrial-based uses of energy [5, 6. Through the capture and reuse of rejected 'waste' energy, it is possible to both increase the operating efficiency of the base cycle as well as reduce Greenhouse Gas (GHG) emissions [5].

The work in this article examines the design and operation of a new, smallscale engine designed to operate from these low-temperature sources using a unique thermodynamic cycle. The Free Piston Expander (FPE) engine operates via modified steam cycle, similar in nature to an Organic Rankine Cycle (ORC), or even direct thermal or heat input. Through focus on a small, millimeter-scale engine, it is possible to use the device across multiple types of thermal sources and applications. For example, a single device may be utilized to scavenge small thermal energy amounts for individual sensor power. By contrast, many FPE engines may be combined in parallel to scavenge thermal energy from larger, industrial size sources.

A microfabricated boiler as well as heat exchangers and other required sys- 
tem components are already under investigation to support the FPE. Thapa et al. investigated a unique MEMS-based boiler using both experiment and computational analysis [7. Heat exchanger research has been conducted using traditional microfabrication materials and methods, like silicon [8]. In this work, the use of capillary channels was the primary means for fluid transport. Relatedly, unique materials and fabrication processes that included electroplated porous nickel [9] or copper [10] have been under development. The option of thermal energy storage for a system of this type has also been investigated using paraffin wax as the storage media [1]. The nature of the FPE and the harvesting system itself allows different working fluids to be substituted for application to a variety of sources and real-world applications.

As part of this scavenging system, the FPE produces useful power output that may be either mechanical or electrical depending on setup and application need. In final form, electrical output may be achieved through the use of piezoelectric materials that flex as a result of piston motion [12 or via linear generator output. The FPE represents a small-scale device with many key dimensions on the order of millimeters. Many components of the device, however, are consistent with common microfabrication techniques and advantages [13.

One of the traditional challenges of power generation on the MEMS or microscale has been the effective management of combustion processes [14]. The feasibility of sustained combustion has been limited by high surface area to volume ratios that exist within miniature combustion systems 15. Wang et al. investigated the miniaturization limit on internal rotary combustion engines [16. Miniaturization of these devices considered losses including mass loss by seals as well as thermal losses. Reduced compression ratio reduced mass loss, or "blow-by," leading to increased efficiency as length scales diminished. The ability to maintain effective operating pressures and low-loss internal sealing 
remains a key challenge.

For this reason, some research has pursued devices and approaches that

60 devices take many forms that have included phase-change actuators that rely on external thermal inputs [17. Pneumatic actuators have also been of interest on the small scale as a further alternative [18]. Phase change steam engines have also been investigated including a micro Newcomen deivce [19]. speed rotational systems that include Brayton or Rankine cycle devices [20. Key among the challenges to this approach is the limitation of decreasing rotor size [21. Below key threshold values, the Brayton cycle and Rankine cycle require alternative designs for typical viscous flows.

70

Rankine cycle efforts have leveraged the advantage of external combustion for the high speed system. System design and modeling [22] as well as fabrication of critical components like microturbopumps [23] have been conducted. Silicon was the material of choice for these efforts, relying on advanced MEMS and microfabrication techniques. Limitations have paralleled those present in fabrication process, external thermal boundary controls, and gap geometry during operation [24]. When applied to a lower temperature energy source, as is the case with many waste heat sources, the use of the Organic Rankine Cycle (ORC) has also been studied on the small scale 25. This approach removes challenges associated with elevated temperature operation, however, remains an engineering challenge for fabrication and production.

Microengines without rotation have included microbubble actuators utilizing capillary channels [26] and membrane-based thermal actuators that produced mechanical power output through internal phase change of a low boiling point 
working fluid. The unique nature of this membrane-type design was further studied for operation approaching the Carnot cycle [27.

In experimental efforts, the ability of the membrane-based actuator to operate at resonant frequency generated a unique working cycle as a result [28]. The general form of these devices utilized one lower membrane with thickness on the order of $1 \mu \mathrm{m}$ and an upper membrane of similar thickness, separated by a cavity volume about $75 \mu \mathrm{m}$ thick. The cavity was filled with working fluid that boiled at low temperature. External heat was added through the lower membrane to generate liquid to vapor phase change. This resulted in increased device volume. When operated at resonant frequency, the pressure and volume changes inside the cavity rose and fell 90 degrees out of phase, producing the unique thermodynamic engine cycle. This unique engine and process was modeled by Bardaweel et al. [29].

Free Piston Engines have been the subject of research and general application for decades on the large scale and in a variety of applications [30]. These engines traditionally consist of a piston within a bore that is free to move between the cycle top-dead-center (minimum volume) and the bottom-dead-center (maximum volume) without mechanical linkage or constraint that is typical of traditional reciprocating engines. This variable compression ratio feature allows fuel flexibility. Applications have varied on the larger scale with internal combustion as the primary driving force. Mechanical outputs have included hydraulic pressure with the free piston used as a linear motion pump 31] as well as electrical power generation. Unique solutions to valve timing and modeling have been investigated due to the lack of rotating components in the basic FPE design [32].

This lack of rotation has made the FPE of interest to the small-scale because it works around many of the aforementioned micro-power challenges. Further, 
the driving force may be either internal or external combustion. This makes its use attractive for sources like waste heat. Various prior approaches have been executed in both modeling and experimental efforts. Early efforts were presented 115 by Aichlmayr et al. and considered internal combustion in a homogeneous charge compression ignition (HCCI) approach [33, 34. This test reaffirmed the challenge to provide precise control of the combustion environment to limit cold surface quenching and manage heat release even in this non-rotating, smallscale application. More recent work has considered the uses of a catalyst wall within the combustion chamber of a micro-FPE that relies on HCCI [35. This numerical effort showed a basic improvement in energy conversion though a decreasing compression ratio due to advanced ignition versus a non-catalytic design. Indicated power output was also increased via this approach. Bai et al. continued efforts at HCCI modeling and established a unique model that 125 predicted the required kinetic energy of the piston to achieve ignition [36]. As with other combustion-centered free piston work, controlling factors included projected sealing leakage as well as accurate equivalence ratio mixing.

Zhang et al. studied a novel 'swing engine' piston approach that moved the free piston in an arc 37. Free Piston Stirling Engines (FPSE) have also been considered and modeled with respect to scaling and operation [38]. Among the opportunities for this design was increased power density, however, energy loss through heat as well as sealing within the engine were among the challenges. Additional approaches include replacing the traditional piston-cylinder assembly with a compliant architecture to facilitate the free-piston motion 39. In this 135 regard, nonlinear lumped parameter engine models and prototypes have been tested to predict engine dynamics and engine performance [40, 41].

Initial results of an external combustion, low-temperature steam cycle Free Piston Expander (FPE) engine have also been investigated with bore dimen- 
sions on the order of a few millimeters [42]. A laser-vibrometer was used to track piston position in the bore as a function of forcing pressure. The natural frequency of the device was approximately $33 \mathrm{~Hz}$.

The past decades have seen continuous progress and the inherent challenges of power production on the small-scale. In the field of engines, these have included the noted challenges to combustion efficiency and quenching, high speed rotation, sealing, and fabrication itself. There remains significant opportunity, however, to perform research on power generation that is both free of rotation and operational from low-grade energy sources. Recent analysis has indicated that the general use of volumetric expanders shows more significant promise than other approaches due to the low flow rates and low grade heat conditions associated with these sources [43. In particular, analysis by Imran et al. indicated Free Piston Expanders had potential to yield lower leakage and reduced frictional losses versus other volumetric expanders 43 .

With these challenges and opportunity in mind, this work presents a new FPE model that is developed using experimental inputs specifically for use in low temperature waste heat application. High speed rotation is avoided as are the challenges of combustion quenching and combustion sealing.

The work contained in the present study follows the basic FPE dimensions of those established by Champagne and Weiss 42. In the present modeling effort, the FPE is approached as a closed cycle device, dependent on external combustion or direct heat input. If applied to a real-world situation, this would require the use of a so-called 'thermal switch.' This thermal switch would introduce thermal energy to the sealed FPE directly from a waste heat source and without fluid crossing any FPE boundary. These switches have been previously demonstrated in external combustion micro-engine development [44. However, 165 as justified in this work, this approximation from an open to closed system in 
FPE operation allows useful interpretation of the basic FPE operation even if applied to a future open system.

To guide future design and testing efforts, key parameters are studied in this work. Specific parameters include the effects of piston mass, engine loading, and working fluid enthalpy or heat input on the unique FPE operating cycle. These provide key insight into the design of a real small-scale FPE engine that is well suited to the important field of low-temperature waste heat scavenging.

\section{Theory}

The basic design of the Free Piston Expander (FPE) is based on the notion of mechanical or electrical power output from an external thermal source. The FPE operates without reliance on high speed rotation or internal combustion which has been difficult to achieve on the microscale as noted previously. Through a sliding-expander type of mechanical action, it is possible to achieve power output without the rotation required of cycles like the Rankine or Brayton. Figure 1 illustrates the setup and key components of the modeled FPE.

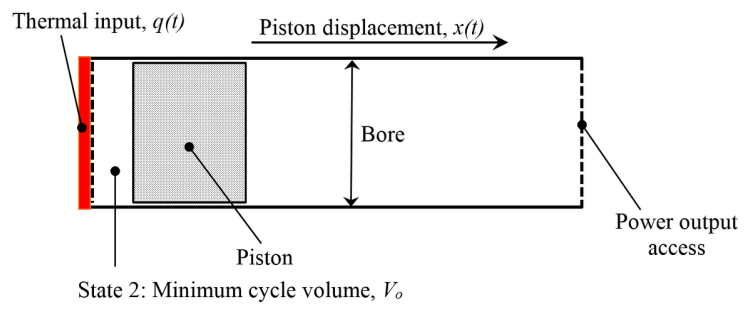

Figure 1: Setup and key components of the FPE

As the piston reacts to increased pressure at the minimum cycle volume (Thermodynamic state 2) it expands. This has the dual effect of reducing the forcing pressure, while increasing the reaction pressure that is building on the other side (nonthermal input side) of the piston. It is this reaction pressure 

external boiler) moves in and out of the FPE as part of its operation.



Figure 2: Schematics sketch of FPE

Based on this basic design, a nonlinear lumped-parameter model was developed to study the expander behavior and performance. Figure 2 shows a schematic diagram of the free piston expander used in the model development. The working fluid of the expander is considered to be a sealed volume $V_{o}$ of vapor in this closed system approach. The basic properties of the fluid are taken to match $3 \mathrm{M}^{T M}$ HFE-7200 working fluid. This provides consistency with parallel experimental efforts and is well suited for low temperature operation given the boiling point of $76^{\circ} \mathrm{C}$. The heat transfer to and from the expander normally associated with super saturated vapor injection and exhaust is modeled by the heat-rate $q(t)$ in and out of expander cavity of volume $V_{o}$ at the left end. The pressure inside the expander cavity oscillates about injection pressure of vapor 
$P_{i}$.

The material enclosing the vapor, the cavity, is represented by a deformable, bellows corrugations. The deformable corrugations of stiffness, $s$, allow horizontal displacement $x(t)$ of the right end of the volume, modeled to have a lump-parameter inertia $m$. The stiffness $s$ is introduced only for computation advantage, hence its numerical value is very low; $s=0.1 \mathrm{~N} / \mathrm{m}$. When the displacement of the mass, $m$, is zero, the volume $V_{o}$ is $V_{o}=L S$, where $L$ is the nominal length and $S$ is the cross-sectional area. The nominal volume $V_{o}$ is numerically equal to $V_{i}$ and $V_{2}$ i.e. $V_{o}=V_{i}=V_{2}$. The movement of the mass, $m$, is impeded by the dampers $b_{f}$ and $b$. These dissipative elements model energy loss to friction and converted to useful work respectively. In addition, a damper with negative damping coefficient $b_{c}\left(b_{c}<0\right)$ is incorporated to model the work done on the expander during the compression stroke.

For a steady state operation, the vapor temperature, $T_{i}+\triangle T(t)$, pressure, $P_{i}+\triangle P(t)$, density, $\rho_{i}+\triangle \rho(t)$, and engine volume, $V_{i}+\triangle V(t)$ undergo cyclic variation, where the nominal and time-varying cyclic components are denoted with the "i" subscript and $\triangle$ respectively.

The nonlinear lumped parameter model of the expander shown in Figure 2 is derived by applying conservation of mass, conservation of energy, an ideal gas model to a moving control volume containing the vapor as working fluid, and Newton's second law for the mass $m$. Mathematical statements of these principles are:

$$
\frac{d M}{d t}=\frac{d}{d t}\left[\left(\rho_{i}+\triangle \rho\right)\left(V_{i}+\triangle V\right)\right]=0
$$




$$
\begin{gathered}
\frac{d}{d t}\left[\left(\rho_{i}+\triangle \rho\right) c_{v}\left(V_{i}+\triangle V\right)\left(T_{i}+\triangle T\right)\right]+\left(P_{i}+\triangle P\right) \frac{d\left(V_{i}+\triangle V\right)}{d t} \\
+h\left(T_{i}-T_{o}+\triangle T\right)=q(t) \\
\rho_{i}+\triangle \rho=\frac{P_{i}+\Delta P}{R\left(T_{i}+\triangle T\right)} \\
\frac{m}{S^{2}} \triangle \ddot{V}+\frac{b+b_{f}+b_{c}}{S^{2}} \Delta \dot{V}+\frac{s}{S^{2}} \triangle V=P_{i}-P_{o}+\triangle P
\end{gathered}
$$

where $M=\rho_{i} V_{i}$ is the mass of vapor in the expander chamber at any one time, $c_{v}$ is the constant volume heat capacity of vapor and is assumed constant over the temperature range, $h$ is a coefficient that models conduction/convection heat losses from the enclosed vapor volume to the outside environment, and $R$ is the mass-specific gas constant of vapor. $T_{o}$ and $P_{o}$ represent ambient temperature and pressure conditions, respectively. The damping coefficient $b_{c}$ is zero for cases where no external work is done on the expander during the compression stroke, and/or for other processes in the expander thermodynamic cycle.

If the heat-rate, $q(t)$, duration of heat addition, $t_{q}$, duration of heat rejection, $t_{c}$, and initial conditions, $\triangle T(0), \triangle P(0)$, and $\triangle V(0)$ are specified, Equations 1 4 constitute a nonlinear model for the determination of the intermediate thermodynamic state variables: $\triangle T(t), \triangle P(t)$, and $\triangle V(t)$. As the heat-rate function $q(t)$ is periodic, it is assumed that the dependent variables $\triangle T(t), \triangle P(t)$, and $\triangle V(t)$ will also be periodic at steady state. The heat-rate function $q(t)$ takes

the form as indicated in Equation 5 and graphically by Figure 3 


$$
q(t)= \begin{cases}q_{H}, & 0<t \leqslant t_{q} \\ 0, & t_{q}<t \leqslant t_{1} \\ q_{o}, & t_{1}<t \leqslant t_{1}+t_{c} \\ 0, & t_{1}+t_{c}<t \leqslant t_{1}+t_{2}\end{cases}
$$

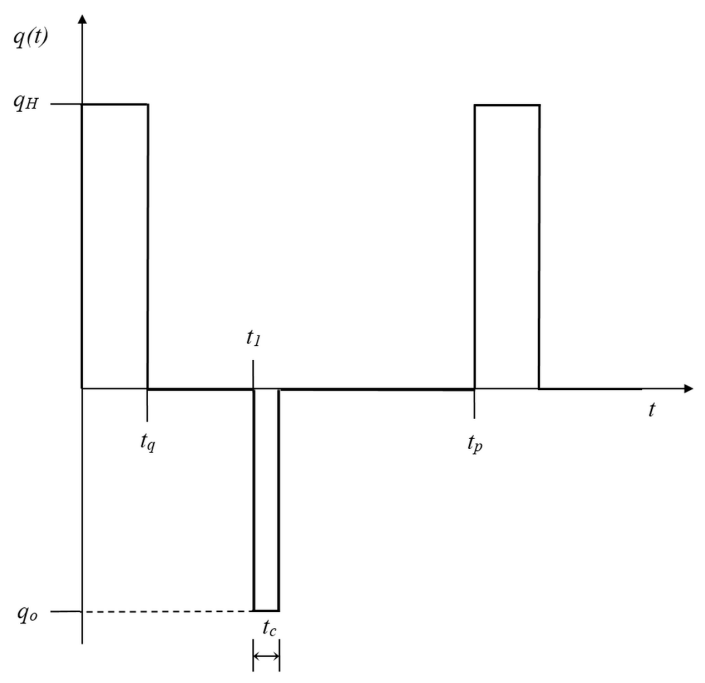

Figure 3: Heat rate function, $q(t)$

The heat-rate function, $q(t)$, consists of finite duration of heating and impulsive cooling. The heating and cooling pulses are of duration $t_{q}$ and $t_{c}\left(t_{c} \rightarrow 0\right)$ respectively. The heating pulse at a constant rate of $q_{H}$ is applied at the end of the compression stroke at $t=0$. At the time $t=t_{1}$, a cooling pulse at a constant rate $q_{o}\left(q_{o}<0\right)$ is then applied. At the time $t=t_{1}+t_{2}=t_{p}$, the cycle is repeated, so that $t_{p}$ is the period of the cyclic heat-rate. This specification for the heat-rate $q(t)$ allows for asymmetry in the cycle, in that the times $t_{1}$ and $t_{2}$, and the magnitude of the heating and cooling pulses, are not necessarily equal.

For the subsequent analysis, a nondimensional scaling was adopted. Equa- 

variables.

$$
\begin{aligned}
\triangle \bar{V} & =\frac{\triangle V}{V_{i}} \\
\triangle \bar{P} & =\frac{\triangle P}{P_{i}} \\
\triangle \bar{T} & =\frac{\triangle T}{T_{i}} \\
\triangle \bar{\rho} & =\frac{\triangle \rho}{\rho_{i}} \\
\bar{q} & =\frac{q}{q_{H}} \\
\bar{t} & =t \omega
\end{aligned}
$$

In Equation 6, $\omega=\sqrt{s_{h} / m}$ is a reference frequency associated with the Helmholtz stiffness $s_{h}=\gamma P_{i} S^{2} / V_{i}$ of the vapor within the expander and the overbar indicates a nondimensional independent or dependent variable. After application of these scales, the nonlinear model [Equations 1- 1 - becomes:

$$
\begin{gathered}
\triangle \bar{\rho}=-\frac{\triangle \bar{V}}{1+\triangle \bar{V}} \\
C \triangle \dot{\bar{T}}=-(1+\triangle \bar{P}) \triangle \dot{\bar{V}}-H\left(1-\frac{T_{o}}{T_{i}}+\triangle \bar{T}\right)+\Gamma \bar{q} \\
\triangle \bar{P}=(1+\triangle \bar{\rho})(1+\triangle \bar{T})-1 \\
\triangle \ddot{\bar{V}}+2\left(\zeta+\zeta_{c}\right) \triangle \dot{\bar{V}}+\Lambda \triangle \bar{V}=\frac{1}{\gamma}\left(1-\frac{P_{o}}{P_{i}}+\triangle \bar{P}\right)
\end{gathered}
$$


where:

$$
\begin{array}{r}
C=\frac{\rho_{i} c_{v} T_{i}}{P_{i}} \\
H=\frac{h T_{i}}{\omega P_{i} V_{i}} \\
\Gamma=\frac{q_{H}}{\omega P_{i} V_{i}} \\
\zeta=\frac{1}{2} \frac{b+b_{f}}{m \omega} \\
\zeta_{c}=\frac{1}{2} \frac{b_{c}}{m \omega} \\
\Lambda=\frac{s}{s_{h}}
\end{array}
$$

For analysis, the set [Equations 7-10] is placed into state space format, the system model, and becomes:

$$
\begin{gathered}
\frac{d \triangle \bar{V}}{d \bar{t}}=\Delta \dot{\bar{V}} \\
\frac{d \dot{\bar{V}}}{d \bar{t}}=-\Lambda \triangle \bar{V}-2\left(\zeta+\zeta_{c}\right) \triangle \dot{\bar{V}}+\frac{1}{\gamma}\left(\frac{1+\triangle \bar{T}}{1+\triangle \bar{V}}-\frac{P_{o}}{P_{i}}\right) \\
\frac{d \triangle \bar{T}}{d \bar{t}}=-\frac{1}{C}\left(\frac{1+\triangle \bar{T}}{1+\triangle \bar{V}}\right) \triangle \dot{\bar{V}}-\frac{H}{C}\left(1-\frac{T_{o}}{T_{i}}+\triangle \bar{T}\right)+\frac{\Gamma}{C} \bar{q}
\end{gathered}
$$

In this format, the state variables are $\triangle \bar{V}, \triangle \dot{\bar{V}}$, and $\triangle \bar{T}$.

To characterize the pressure-volume behavior of the expander in this work, the model [Equations 7]- 10] was integrated numerically. It was assumed that the duration of the heating pulse $t_{q}$ is finite such that $q_{H} t_{q}=E$, where $E$ is the finite energy supplied to the expander per cycle during the heating pulse. In this circumstance the pressure-volume behavior of the expander at steady state takes the form shown in Figure 4. Thermodynamic states 1-2-3-4 on the diagram correspond to the end of impulsive cooling and beginning of the 
compression stroke, end of the compression stroke and beginning of the heating, end of heating and beginning of expansion stroke, and the end of the expansion stroke respectively. The expander volume and the temperature inside it before and after the heat rejection can be computed from Equations 13 and 14 and are given by Equations 15 and 16 .

$$
\begin{gathered}
\Delta \overline{V_{1}}=\Delta \overline{V_{4}} \\
\triangle \overline{T_{1}}=\Delta \overline{T_{4}}+\frac{\Gamma t_{c} \omega}{C}\left(\frac{q_{o}}{q_{H}}\right)
\end{gathered}
$$

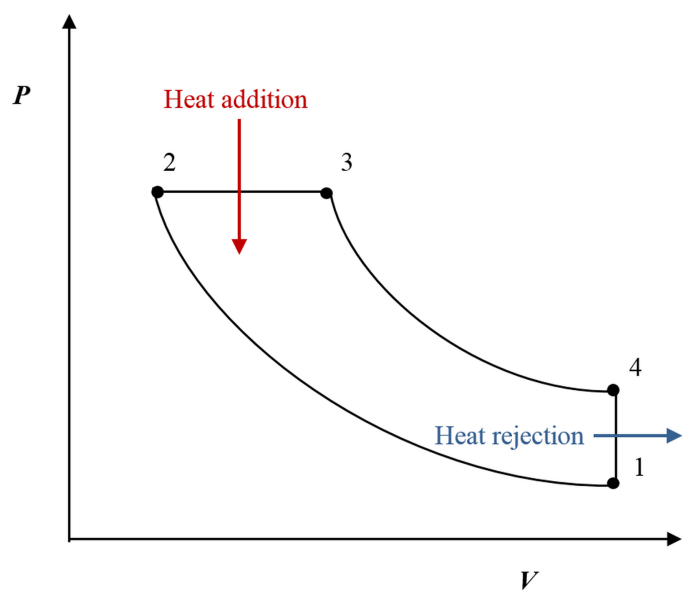

Figure 4: Steady state P-V diagram

It was assumed that the impulsive cooling pulses occur at zero piston velocity, $\Delta \dot{\overline{V_{4}}}=\Delta \dot{\overline{V_{1}}}=0$. The resulting volumes and temperatures $\Delta \overline{V_{4}}, \Delta \overline{T_{4}}$ and $\triangle \overline{V_{2}}, \triangle \overline{T_{2}}$ from expansion and compression processes $3-4$ and 1-2 were computed by numerically integrating [Equations 12 - 14 from the initial con275 ditions $\triangle \overline{V_{3}}, \triangle \overline{T_{3}}, \triangle \dot{\overline{V_{3}}}$ and $\triangle \overline{V_{1}}, \triangle \overline{T_{1}}, \Delta \dot{V_{1}}=0$. Similarly, the resulting volume $\triangle \overline{V_{3}}$ and temperature $\triangle \overline{T_{3}}$ from heat addition process 2-3 were com- 
puted by numerically integrating [Equations 12 - 14 from the initial condition $\triangle \overline{V_{2}}=0, \triangle \overline{T_{2}}=0, \triangle \overline{V_{2}}=0$.

The system [Equations 12-14 can only reach steady state when the proper cooling pulse magnitude $q_{o} / q_{H}$ and compression work modeled by damper $b_{c}$ are chosen for cycle integration. Improper choice of $q_{o} / q_{H}$ and $b_{c}$ would lead to monotonically drifting state variables $\triangle \bar{V}$ and $\triangle \bar{T}$ precluding periodic behavior. To determine the steady-state behavior of the expander $q_{H}, \triangle \overline{V_{2}}=0, \triangle \overline{T_{2}}=$ 0 , and $\triangle \overline{P_{2}}=0$ were specified, the ratio $q_{o} / q_{H}$ and damping coefficient $b_{c}$ were chosen, and integration was started from the quiescent initial state of $\Delta \overline{V_{2}}=\Delta \dot{V_{2}}=\Delta \overline{T_{2}}=0$. An example integration illustrating the first cycle in a transient dwell-up from this initial state is shown in Figure 5.

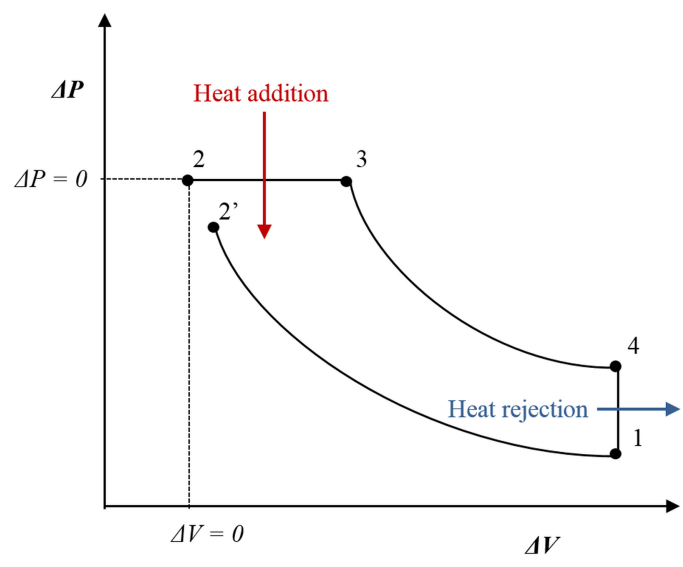

Figure 5: Transient state P-V diagram

First, temperature and volume were computed for the thermodynamic cycle 1-2-3-4-1 as explained below and later Equations 7 and 9 were used to calculate 290 corresponding pressure. The volume $\triangle \overline{V_{3}}$ and temperature $\Delta \overline{T_{3}}$ were obtained by integrating the set [Equations 12 - 14 from the initial condition $\triangle \overline{V_{2}}=$ $\Delta \dot{V_{2}}=\triangle \overline{T_{2}}=0$ until a time $\overline{t_{q}}$. The volume $\triangle \overline{V_{4}}$ and temperature $\triangle \overline{T_{4}}$ were 
obtained by integrating the set [Equations 12 - 14 from the initial conditions $\triangle \overline{V_{3}}, \triangle \overline{T_{3}}, \Delta \dot{\overline{V_{3}}}$ until a time, $\overline{t_{1}}$, such that $\Delta \dot{\overline{V_{4}}}=0$. Note that the time, $\overline{t_{1}}$ is not known 'a priori', and is determined during the integration. Then, the volume $\triangle \overline{V_{1}}$ and temperature $\triangle \overline{T_{1}}$ were determined from Equations 15 and 16 . Finally, a volume $\triangle \overline{V_{2^{\prime}}}$ and $\triangle \overline{T_{2^{\prime}}}$ were obtained by integrating the set [Equations 12 14 from the initial conditions $\triangle \overline{V_{1}}, \triangle \overline{T_{1}}, \triangle \dot{V_{1}}=0$ until a time, $\overline{t_{2}}$, such that $\Delta \dot{\overline{V_{2^{\prime}}}}=0$. Like the time of expansion, $\overline{t_{1}}-\overline{t_{q}}$, the time of compression, $\overline{t_{2}}$, is not 300 known 'a priori', and is determined by the conditional integration. Since the system undergoes a transient phase, the cycle is not closed and $\triangle \overline{V_{2}} \neq \Delta \overline{V_{2^{\prime}}}$ and $\triangle \overline{T_{2}} \neq \triangle \overline{T_{2^{\prime}}}$ However, if the process is repeated, and the proper ratio $q_{o} / q_{H}$ and damping coefficient, $b_{c}$, are chosen, the cycle eventually reaches steady state, the cycle closes, and $\triangle \overline{V_{2}}=\Delta \overline{V_{2^{\prime}}}$ and $\triangle \overline{T_{2}}=\Delta \overline{T_{2^{\prime}}}$. The integration stops when the equality has been satisfied to a specified tolerance. In practice, a functionminimization computational procedure was used, which determined the proper choice of $q_{o} / q_{H}$ and $b_{c}$ for a specified $q_{H}$ that would result in a steady-state periodic cycle.

\section{Results and Discussion}

The small-scale free piston expander shown in Figures 1 and 2 is modeled with $h=0 \mathrm{~W} / \mathrm{K}, b_{f}=0 \mathrm{N-s} / \mathrm{m}, b=3 \mathrm{~N}-\mathrm{s} / \mathrm{m}, V_{o}=56.5 \mathrm{~mm}^{3}(4.8 \mathrm{~mm}$ in diameter and $3.2 \mathrm{~mm}$ of nominal length), $s=0.1 \mathrm{~N} / \mathrm{m}$, and $m=0.34 \mathrm{~g}$ treated as the reference case. Standard temperature and pressure conditions of $T_{o}=298 \mathrm{~K}$ and $P_{o}=101 \mathrm{kPa}$ are chosen for the ambient state. The injection 315 temperature and pressure of the working fluid are selected to be $T_{i}=373 \mathrm{~K}$ and $P_{i}=105 \mathrm{kPa}$ respectively. The properties of working fluid used are $R=24.6$ $\mathrm{J} / \mathrm{kg}-\mathrm{K}, \rho=11.4 \mathrm{~kg} / \mathrm{m}^{3}, c_{v}=1069 \mathrm{~J} / \mathrm{kg}-\mathrm{K}$, and $c=96.9 \mathrm{~m} / \mathrm{s}$. These values are based on real $3 \mathrm{M}^{T M}$ HFE-7200 properties. The nonlinear model [Equations 7 - 
10 is used to predict the performance of the free piston expander.

Figure 6 shows a plot of expander pressure and volume for one thermodynamic cycle 1-2-3-4-1. It is obtained using the nonlinear model [Equations 710 for a steady state operation by supplying heat at a rate $q_{H}=2 \mathrm{~W}$ for a duration $t_{q}=1.9 \mathrm{~ms}$ at the end of the compression process 1-2 to the reference 325 case. The ratio $q_{o} / q_{H}=-0.99$ and $b_{c}=-3.27 \mathrm{~N}-\mathrm{s} / \mathrm{m}$ are required for steady state operation of the nonlinear model. The figure shows that the pressure inside the expander throughout the heat addition process remains nearly constant at about $105 \mathrm{kPa}$, indicating a constant pressure process. Following the heat addition process 2-3, the expander expands isentropically $3-4$ to about $59.5 \mathrm{~mm}^{3}$. 330 Next, the heat rejection process $4-1$ takes place instantaneously by interacting with the surroundings. Finally, the expander undergoes isentropic compression 1-2 due to external work resulting in a minimum cycle volume $V_{2}=V_{o}=56.5$ $\mathrm{mm}^{3}$.

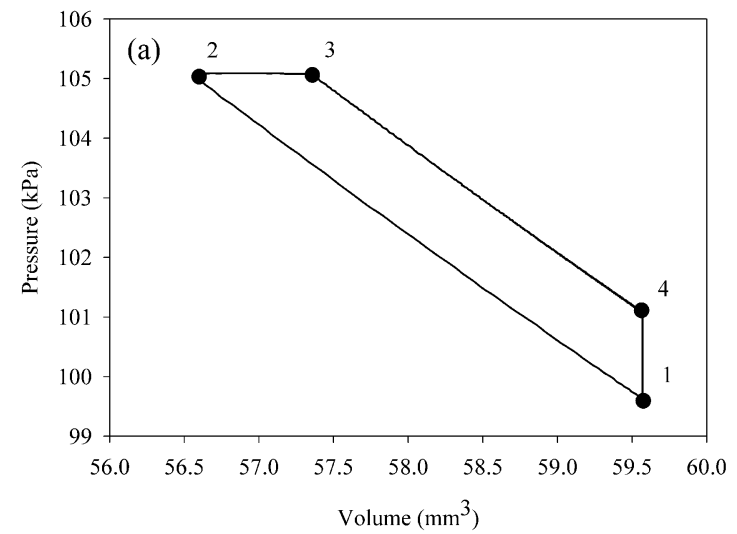

Figure 6: P-V diagram for the reference expander for input heat-rate $q_{H}=2 \mathrm{~W}$

To determine the work done per cycle, a numerical integration of the P-V 

$3.9 \mu \mathrm{J}$. From the figure, it has been found that the work done by the expander during the overall expansion and compression processes 2-4 and 1-2 are 309.2 $\mu \mathrm{J}$ and $-305.3 \mu \mathrm{J}$ respectively. The corresponding expander thermal efficiency for one thermodynamic cycle was calculated to be $0.1 \%$.

Figure 7 shows a plot of expander temperature and volume for one thermodynamic cycle. It is obtained for the same operating conditions used for generating P-V diagram in Figure 6] It is evident from the figure that the constant rate heat addition process 2-3 is not a constant temperature process.

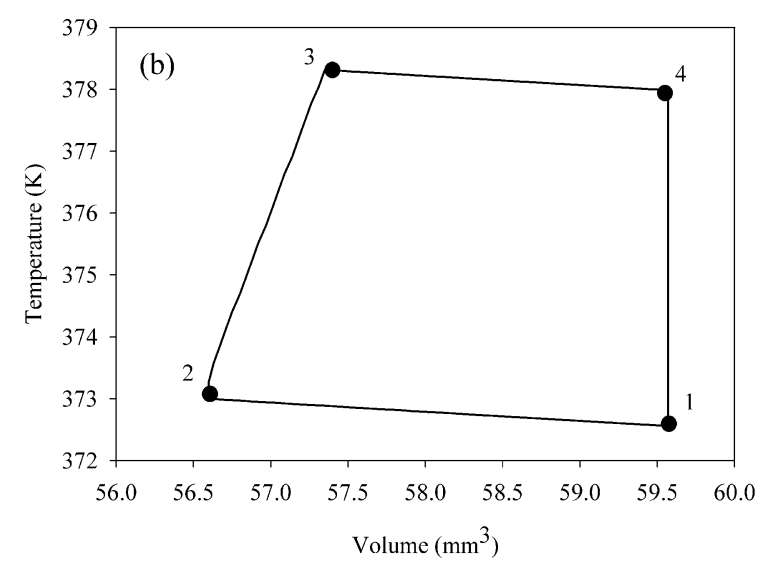

Figure 7: T-V diagram for the reference expander for input heat-rate $q_{H}=2 \mathrm{~W}$

Though the expansion and compression processes 3-4 and 1-2 are isentropic, the temperature change in them are small $\triangle \mathrm{T}=0.5 \mathrm{~K}$ due to the small change in expander cavity volume. However, during the heat rejection process 4-1, the temperature drops significantly by $\triangle \mathrm{T}=5.5 \mathrm{~K}$. From the figure, the temperature inside the expander over one thermodynamic cycle varies between 372.5 $\mathrm{K}$ and $378.5 \mathrm{~K}$ indicating that the working fluid is always superheated based 350 on $3 \mathrm{M}^{T M}$ provided thermodynamic tables. Ideal gas behavior is assumed as a 
result, as has been successful in other modeling approaches [45].

\subsection{Expander Performance}

The expander performance is evaluated in terms of thermal efficiency and power output. Output power from the expander is computed assuming that the only dissipation mechanism present is the energy conversion damping $b$ (also called loading). The nonlinear model [Equations 7]- 10 is used to study the effects of design and operating parameters such as enthalpy of the working fluid, piston mass, and load on the expander performance.

\subsubsection{Enthalpy of the working fluid}

360

The enthalpy of the working fluid depends on its mass, thermodynamic properties, and thermodynamic state variables. Since the thermodynamic properties and mass of the closed system remain unchanged, the enthalpy depends solely on the thermodynamic state variables: temperature and pressure. Studying the effect of enthalpy provides insight in to injection temperature and pressure of the working fluid for achieving greater expander performance. Therefore to study the effect of enthalpy on expander performance, the injection temperature and pressure are varied. The temperature variation is accomplished by adding heat at the end of the compression process 1-2, while the injection pressure is varied by setting the expander pressure at state $2, P_{2}$. The heat addition to the expander depends on input heat-rate and duration of heat addition process 2-3. For our analysis, only heat-rate is varied, while the duration of heat addition is kept constant i.e. $t_{q}=1.9 \mathrm{~ms}$.

Input Heat Rate. The effect of working fluid injection temperature on the expander performance is evaluated by adding heat at the end of the compression process $1-2$. For a fixed duration of heat addition $t_{q}=1.9 \mathrm{~ms}$, the input heatrate $q_{H}$ is varied. Figure 8 , shows the expander behavior in the form of a P-V 
diagram plotted for various input heat-rates $q_{H}=1,2$, and $3 \mathrm{~W}$, piston mass $m=0.34 \mathrm{~g}$, load $b=3 \mathrm{~N}-\mathrm{s} / \mathrm{m}$, and injection pressure $P_{i}=P_{2}=105 \mathrm{kPa}$. It can be observed that for a constant input heat-rate $q_{H}=2 \mathrm{~W}$, the heat addition process 2-3 models a constant pressure process. However, for higher or lower input heat-rates the model departs from constant pressure process. This indicates that there exists a computational domain in which the closed cycle, constant heat-rate modeling approach models a constant pressure cycle. Also, it can be observed that for increasing input heat-rate the P-V diagram expands, implying an increase in work output.

For an increase in input heat-rate from $q_{H}=1$ to $q_{H}=2 \mathrm{~W}$, the work output increased from 1.6 to $3.9 \mu \mathrm{J} /$ cycle which translates to an increase in efficiency from $0.09 \%$ to $0.13 \%$. On the other hand, the operating frequency of the expander decreased from $f=39.8 \mathrm{~Hz}$ to $f=38.6 \mathrm{~Hz}$ owing to the resulting 390 larger stroke lengths. Despite the reduction of operating frequency, the output power increased from $66 \mu \mathrm{W}$ to $150 \mu \mathrm{W}$ due to the significant rise in work output per cycle. Therefore, it is desired to operate the expander with higher input heat-rate to achieve higher efficiency and output power. However, in practice there exists an upper limit on the input heat-rate determined by enthalpy of 395 the vapor or working fluid as well as the actual real-world heat source itself.

Injection pressure. As previously noted, injection pressure of the working fluid also determines the enthalpy of the working fluid. To study the effects of enthalpy, the injection pressure is varied while maintaining input heat-rate constant. For this, the expander pressure at state 2 is set to be equal to the injection pressure $P_{i}$. Figure 9 shows a P-V diagram generated for three injection pressures; $P_{i}=105,110$, and $115 \mathrm{kPa}$, while maintaining the input heat-rate $q_{H}$ $=2 \mathrm{~W}$, input heat $E=3.8 \mathrm{~mJ} /$ cycle, piston mass $m=0.34 \mathrm{~g}$, and load $b=$ $3 \mathrm{~N}-\mathrm{s} / \mathrm{m}$. From the figure, despite same minimum volume $V_{2}$, an increase in 


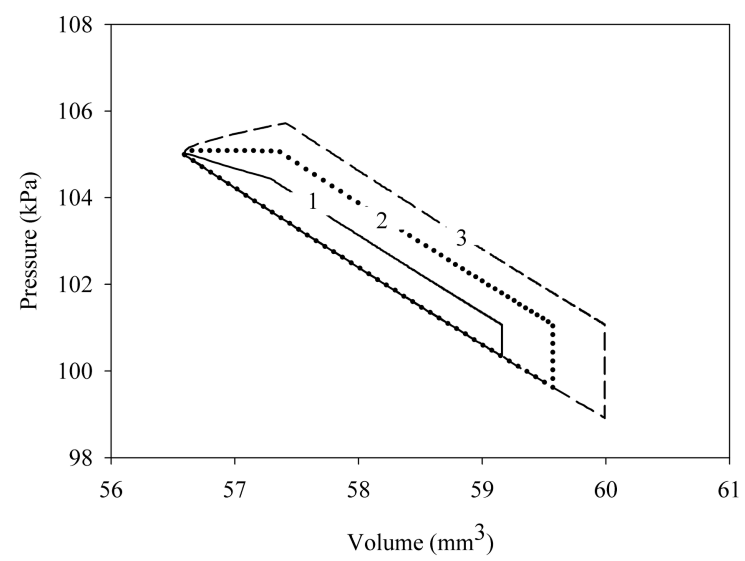

Figure 8: P-V diagram for the reference expander and varying input heat-rates $q_{H}=1,2$, and $3 \mathrm{~W}$.

injection pressure increased the swept volume $V_{2} \rightarrow V_{4}$. For instance, the swept volume $V_{2} \rightarrow V_{4}$ for $P_{i}=105 \mathrm{kPa}$ is about $3 \mathrm{~mm}^{3}$, while for $P_{i}=110 \mathrm{kPa}$ the swept volume $V_{2} \rightarrow V_{4}$ is about $6 \mathrm{~mm}^{3}$. Though the heat input for both conditions is maintained at $E=3.8 \mathrm{~mJ} /$ cycle there is an increase in output work. This implies that higher injection pressure is desirable for higher output work. It is clear from the figure that the constant input heat-rate departs from constant pressure cycle for higher injection pressure. It is also worth noting that the expander pressure equilibrates with ambient pressure at the end of the expansion process 3-4 irrespective of the injection pressure $P_{i}$. The nonlinear model predicted that the operating frequency decreases from $f=38.6 \mathrm{~Hz}$ to $f=$ $33.2 \mathrm{~Hz}$ for an increase in injection pressure $P_{i}$ from $105 \mathrm{kPa}$ to $110 \mathrm{kPa}$. This 415 means that the model predicts a softening behavior of the expander system. A similar trend was noted in direct study of the input heat rate as discussed previously. 


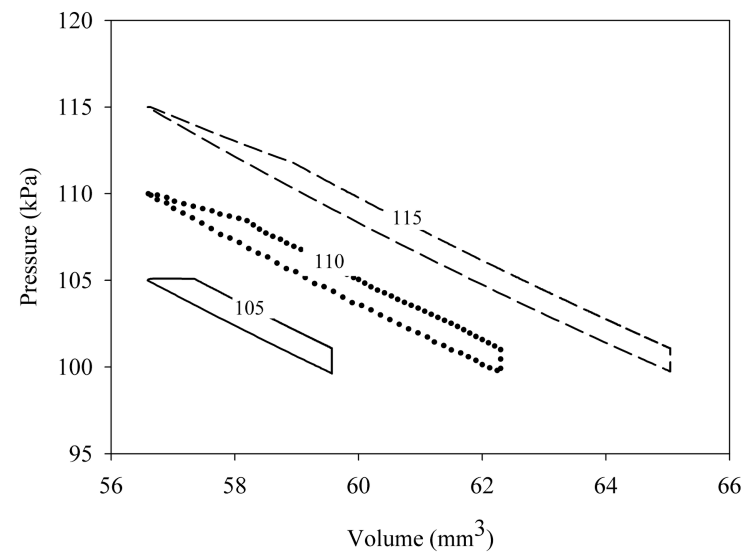

Figure 9: P-V diagram for the reference expander for input heat-rate $q_{H}=2 \mathrm{~W}$ and varying injection pressures $P_{i}=105,110$, and $115 \mathrm{kPa}$.

\subsubsection{Load}

In the nonlinear model [Equations 7]-10], the load $b$ models the work output or useful work while the frictional load $b_{f}$ models friction loss. In this study, the frictional damping $b_{f}$ is assumed to be negligible. This is based on prior experimental efforts that monitored the effect of various lubricants on a small-scale piston / bore assembly as well as the piston cyclic response [42. These results allowed determination of a frictional damping coefficient to be approximately 425 .014 $\frac{N-s}{m}$, well below the minimum engine loadings considered in this present work.

The effect of load on the engine performance is captured in the form of a P-V diagram shown in Figure 10

The P-V diagram shown in Figure 10 is generated for three loads, $b=3,4$, ${ }_{430}$ and $5 \mathrm{~N}-\mathrm{s} / \mathrm{m}$ at constant input heat-rate $q_{H}=2 \mathrm{~W}$ and injection pressure $P_{i}$ $=105 \mathrm{kPa}$. It can be observed that when the load on the expander is increased from $b=3 \mathrm{~N}-\mathrm{s} / \mathrm{m}$ to $b=5 \mathrm{~N}-\mathrm{s} / \mathrm{m}$ the heat addition process 2-3 begins to depart from a constant pressure process. This can be explained via the ideal gas law 


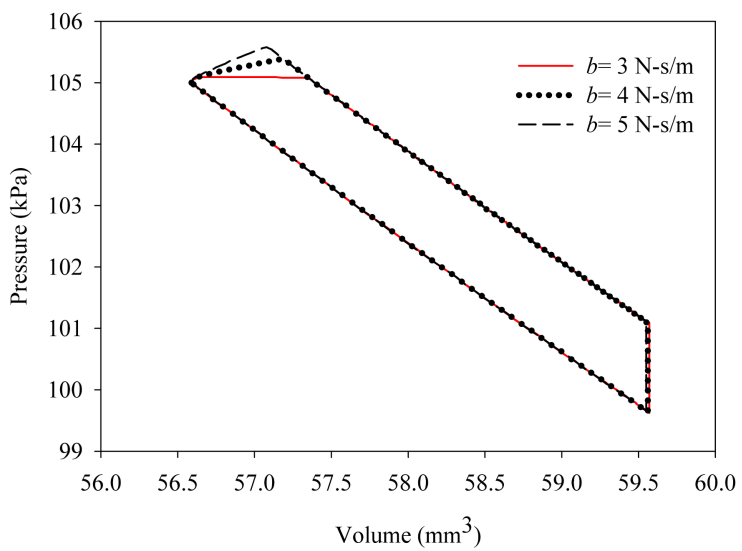

Figure 10: $\mathrm{P}-\mathrm{V}$ diagram for the reference expander for input heat-rate $q_{H}=2 \mathrm{~W}$ and varying loads $\mathrm{b}=3,4$, and $5 \mathrm{~N}-\mathrm{s} / \mathrm{m}$

and treating the expander as a spring-mass-damper system.

For a fixed injection pressure $P_{i}$, input heat-rate $q_{H}$, and higher loads $b$, the piston velocity decreases due to the increased damping on the expander; thus resulting in a positive rate of change of pressure. Based on a spring-massdamper system analogy it is assumed that the stroke length gets smaller for higher load $b$. Despite increasing load $b$, the expander stroke length remains nearly constant. This is due to the fact that higher load in turn results in higher expander pressure at state 3 that can push the piston further outward (thermodynamic state 4 ).

It is evident from the figure, the increase in load on the expander from $b=3$ $\mathrm{N}-\mathrm{s} / \mathrm{m}$ to $b=5 \mathrm{~N}-\mathrm{s} / \mathrm{m}$ did not significantly increase the area enclosed by the P-V diagram. The increased area during the heat addition process 2-3 is nullified by the decrease during the expansion process 3-4. However, a significant change in natural frequency of the expander was observed for different loads $b=3,4$, and $5 \mathrm{~N}-\mathrm{s} / \mathrm{m}$. The natural frequency dropped from $38.6 \mathrm{~Hz}$ for $b=3 \mathrm{~N}-\mathrm{s} / \mathrm{m}$ to 27.1 $\mathrm{Hz}$ for $b=5 \mathrm{~N}-\mathrm{s} / \mathrm{m}$. This behavior was anticipated and can be explained along 
in the presence of a damper given by the equation $\omega_{d}=\omega_{n} \sqrt{1-\zeta^{2}}$, where $\omega_{n}$ is the natural frequency of the oscillator, $\omega_{d}$ is the damped natural frequency of the oscillator, and $\zeta$ is the damping ratio. Table 1 presents expander performance for various loads and fixed heat input $E=3.8 \mathrm{~mJ} /$ cycle.

\begin{tabular}{|c|c|c|c|}
\hline $\begin{array}{c}\text { Load } \\
(\mathrm{N}-\mathrm{s} / \mathrm{m})\end{array}$ & $\begin{array}{c}\text { Efficiency } \\
(\%)\end{array}$ & $\begin{array}{c}\text { Operating Freq. } \\
(\mathrm{Hz})\end{array}$ & $\begin{array}{c}\text { Output Power } \\
(\mathrm{mW})\end{array}$ \\
\hline 3 & 0.103 & 38.6 & 15.1 \\
4 & 0.106 & 31.6 & 12.7 \\
5 & 0.108 & 27.1 & 11.1 \\
\hline
\end{tabular}

Table 1: Output power for various loading conditions for fixed input heat $E=3.8 \mathrm{~mJ} / \mathrm{cycle}$

\subsubsection{Piston Mass}

The effect of piston mass on the engine performance is crucial as it is one of the key factors that dictates expander operating frequency. It is presumed that a smaller piston mass will result in higher natural or operating frequency that can result in higher output power. Also, studying the effect of piston mass can help identify an optimum piston size and type of material.

Figures 11 and 12 show a P-V diagram and volume-velocity diagram for three piston masses $m=0.34,1.7$, and $3.4 \mathrm{~g}$, input pressure $P_{i}=105 \mathrm{kPa}$, load $b=1 \mathrm{~N}-\mathrm{s} / \mathrm{m}$, heat addition time $t_{q}=1.9 \mathrm{~ms}$, and input heat $E=10 \mathrm{~mJ} / \mathrm{cycle}$, respectively. It is evident from the figure that upon increasing the piston mass from $0.34 \mathrm{~g}$ to $1.7 \mathrm{~g}$, the inertia effect on the engine behavior becomes evident. Though higher piston mass implies higher resistance to motion, the pistons with higher mass unusually have longer stroke lengths. This can be understood by analyzing the expander total energy as discussed below.

The expander can be assumed to be a spring-mass-damper system whose total energy is the sum of thermal energy in the expander cavity, dissipative energy in the form of output work, kinetic energy of the piston, and potential 




Figure 11: P-V diagram for load $b=1 \mathrm{~N}-\mathrm{s} / \mathrm{m}$, input heat $E=10 \mathrm{~mJ} /$ cycle, and varying mass $m$

energy stored in the spring. For analysis of piston mass effects directly, the change in thermal energy of the working fluid and the dissipative energy are assumed to be negligible. Therefore, the total energy comprises of kinetic and 475 potential energies. Since the heat addition per cycle $E=10 \mathrm{~mJ} /$ cycle is constant, the total energy is distributed between the piston and the spring. It is known that the kinetic energy in the piston assists the piston to travel a longer distance due higher momentum, while the potential energy in the spring retards the piston travel due to its opposing nature resulting in shorter stroke lengths. Therefore, for higher piston masses the kinetic energy dominates the potential energy resulting in larger stroke lengths and vice-versa. The effect of the piston mass on the operating frequency is schematically represented in Figure 13 . The increasing piston mass resulted in decreasing natural frequency $f$ and can be explained using the relation $f=\sqrt{s / m}$, where $s$ is the stiffness of spring and $m$ is the piston mass. Such similar trends have been observed by Weiss [12].

The effect of the piston mass on the expander efficiency and output power for a fixed input heat $E=10 \mathrm{~mJ} /$ cycle, load $b=1 \mathrm{~N}-\mathrm{s} / \mathrm{m}$, and injection pressure $P_{i}$ 


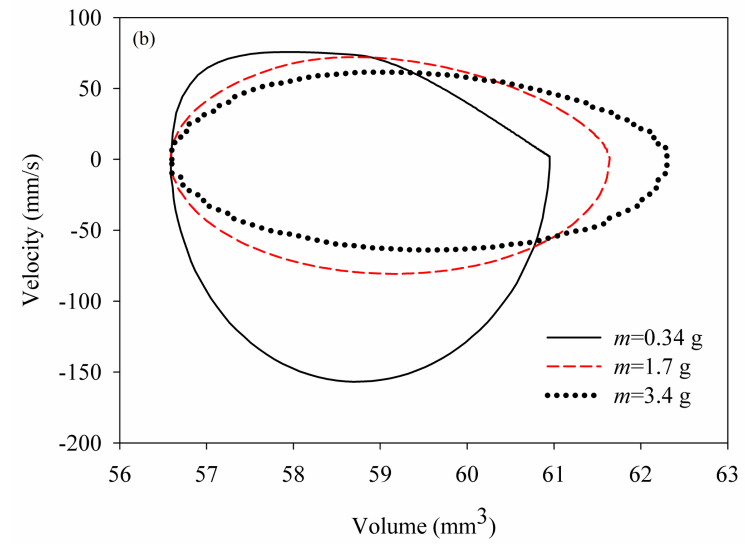

Figure 12: Volume-velocity diagram for load $b=1 \mathrm{~N}-\mathrm{s} / \mathrm{m}$, input heat $E=10 \mathrm{~mJ} / \mathrm{cycle}$, and varying mass $m$

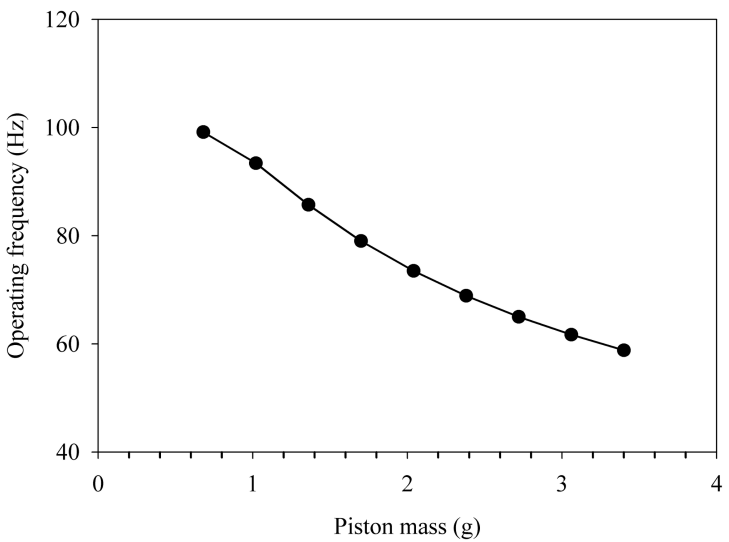

Figure 13: Operating frequency dependency on mass $\mathrm{m}$ for fixed input heat $E=10 \mathrm{~mJ} /$ cycle and load $b=1 \mathrm{~N}-\mathrm{s} / \mathrm{m}$

$=105 \mathrm{kPa}$ is schematically represented in Figure 14 From Figure 14 it is clear that the efficiency steadily rises while the output power rises and then drops with the increase in piston mass. The input heat $E=10 \mathrm{~mJ} /$ cycle and duration of heat input $t_{q}=1.9 \mathrm{~ms}$ were held constant, and piston mass $m$ was varied to find the optimum output power and efficiency. The results presented in Table 
2 show that the piston mass $m=1.02 \mathrm{~g}$ produces a maximum output power of $148 \mathrm{~mW}$ at an efficiency of $0.16 \%$. Despite the trend that lower piston masses in the range $m \in(0.34-2.04 \mathrm{~g})$ produce higher output power, higher efficiencies $\eta>0.2 \%$ are achieved for piston masses greater than $m>2.38 \mathrm{~g}$. Given the low temperature operating conditions imposed on the FPE, it is recommended to use higher piston masses to achieve respectable thermal efficiencies.

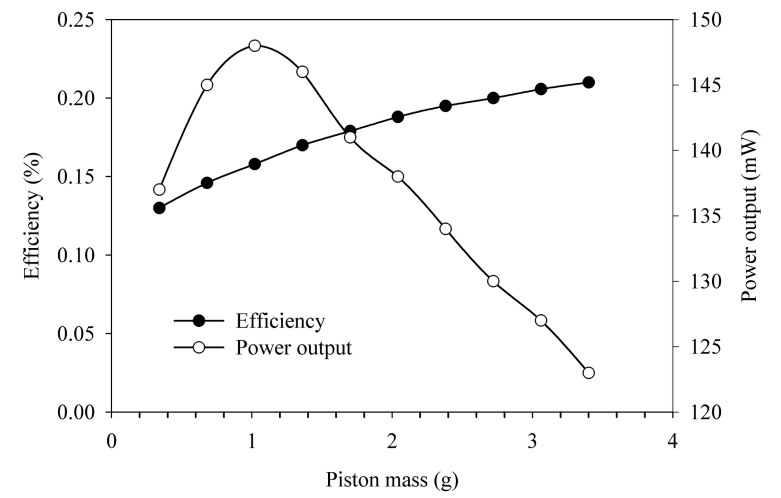

Figure 14: Efficiency and output power dependency on piston mass $m$

\begin{tabular}{|c|c|c|c|}
\hline $\begin{array}{c}\text { Piston Mass } \\
(\mathrm{g})\end{array}$ & $\begin{array}{c}\text { Efficiency } \\
(\%)\end{array}$ & $\begin{array}{c}\text { Operating Freq. } \\
(\mathrm{Hz})\end{array}$ & $\begin{array}{c}\text { Output Power } \\
(\mathrm{mW})\end{array}$ \\
\hline 0.34 & 0.13 & 102.1 & 137 \\
0.68 & 0.15 & 99.2 & 145 \\
1.02 & 0.16 & 93.4 & 148 \\
1.36 & 0.17 & 85.7 & 146 \\
1.70 & 0.18 & 79.0 & 141 \\
2.04 & 0.19 & 73.5 & 138 \\
2.38 & 0.20 & 68.9 & 134 \\
2.72 & 0.20 & 65.0 & 130 \\
3.06 & 0.21 & 61.7 & 127 \\
3.40 & 0.21 & 58.8 & 123 \\
\hline
\end{tabular}

Table 2: Output power for various loading conditions for fixed input heat $E=3.8 \mathrm{~mJ} /$ cycle 


\section{Conclusions}

The ability to find new uses for low temperature energy sources like waste heat continues to grow in importance due to factors like fossil fuel use and coupled greenhouse gas emissions. This work examined the operating cycle and characteristics of a new, small-scale Free Piston Expander (FPE) engine designed to operate from these sources. The basic analysis considered a closed cycle operation of the FPE with suitably low thermal inputs and dimensions on the order of several millimeters.

A new, nonlinear lumped-parameter model was created to provide initial evaluation of the FPE and the FPE cycle. The essential expander behavior and performance was captured through this approach. The effects of several key system design and operating parameters were considered. Of specific interest were the cycle power output, projected efficiency, and critical design parameters. Variables considered included various piston masses, external loads, and input heat-rates.

There were several key results. First, a new operating cycle was indicated that was similar in form to a constant pressure cycle but highly dependent on operating inputs. These included the input heat-rate, duration of heat input, and load on the expander itself. Second, the nature of the piston being driven by surrounding pressure balances gave the FPE the potential for a variety of closed cycle loops and shapes. Third, indicated efficiencies were low and ranged from about 0.1 to $0.2 \%$. This was driven by the assumed low temperature gradient driving FPE operation as guided by waste thermal energy itself. Fourth, the efficiency was shown to be additionally dependent on design parameters, most notably the piston mass and heat input rate. Increased heat input had the effect of both reducing the FPE operating frequency while increasing the total power 25 output. This was due to the increased stroke length resulting from increased 
energy input. The resulting stroke length increase also resulted in an expanded work output per cycle, however, a reduction in the frequency. Despite this operating frequency reduction, the expanded work output from the pressurevolume diagram produced a total increase in power output of about $127 \%$.

Despite the low efficiency result, good scope for improving basic design and performance output was generated from the analysis. Additional gains in power output and efficiency are easily achieved through operation of the engines in parallel or series as well as an increased operating temperature gradient specific to any targeted real world application. This work provides the basis for further nonlinear modeling in an open cycle approach. This work also provides valuable insight into the operation and characteristics of an experimental FPE, designed to operate in a small-scale thermal scavenging application.

\section{Acknowledgments}

The authors gratefully acknowledge the support of this work by the NSF via 540 Grant No. ECCS-1053729 


\section{Nomenclature}

\begin{tabular}{|c|c|}
\hline$b$ & : Load $(\mathrm{N}-\mathrm{s} / \mathrm{m})$ \\
\hline$b_{c}$ & : Damping coefficient for external work on the engine $(\mathrm{N}-\mathrm{s} / \mathrm{m})$ \\
\hline$b_{f}$ & : Frictional damper $(\mathrm{N}-\mathrm{s} / \mathrm{m})$ \\
\hline$c$ & : Speed of sound $(\mathrm{m} / \mathrm{s})$ \\
\hline$C$ & : Nondimensional heat capacity of vapor \\
\hline$c_{v}$ & : Constant volume heat capacity of vapor $(\mathrm{J} / \mathrm{kg}-\mathrm{K})$ \\
\hline$E$ & : Finite energy supplied to the expander per cycle $(\mathrm{mJ} /$ cycle $)$ \\
\hline$f$ & : Engine operating frequency $(\mathrm{Hz})$ \\
\hline$h$ & : Heat loss coefficient $(\mathrm{W} / \mathrm{K})$ \\
\hline$H$ & : Nondimensional heat loss coefficient \\
\hline$L$ & : Cylinder nominal length $(\mathrm{mm})$ \\
\hline$m$ & : Piston mass (g) \\
\hline$M$ & : Working fluid mass (kg) \\
\hline$P$ & : Pressure $(\mathrm{kPa})$ \\
\hline$P_{i}$ & : Injection pressure of vapor $(\mathrm{kPa})$ \\
\hline$q(t)$ & : Heat-rate $(\mathrm{W})$ \\
\hline$q_{H}$ & : Heating pulse at constant rate $(\mathrm{W})$ \\
\hline$q_{o}$ & : Cooling pulse at constant rate $(\mathrm{W})$ \\
\hline$R$ & : Mass-specific gas constant of vapor $(\mathrm{J} / \mathrm{kg}-\mathrm{K})$ \\
\hline$s$ & : Stiffness of deformable corrugations $(\mathrm{N} / \mathrm{m})$ \\
\hline$S$ & : Cylinder cross-sectional area $\left(\mathrm{mm}^{2}\right)$ \\
\hline$s_{h}$ & : Helmholtz stiffness $(\mathrm{N} / \mathrm{m})$ \\
\hline$t$ & : time $(\mathrm{s})$ \\
\hline$T$ & : Temperature $(\mathrm{K})$ \\
\hline$t_{1}$ & : Time at end of expansion process (ms) \\
\hline$t_{2}$ & : Duration of compression process (ms) \\
\hline$t_{c}$ & : Duration of heat rejection (ms) \\
\hline$t_{p}$ & : Engine cycle time period (ms) \\
\hline$t_{q}$ & : Duration of heat addition (ms) \\
\hline$V$ & : Volume $\left(\mathrm{mm}^{3}\right)$ \\
\hline$V_{0}$ & : Expander nominal cavity volume $\left(\mathrm{mm}^{3}\right)$ \\
\hline$x(t)$ & : Piston displacement $(\mathrm{mm})$ \\
\hline$\gamma$ & : Ratio of specific heats \\
\hline$\triangle$ & : Time-varying cyclic component \\
\hline$\zeta$ & : Damping ratio \\
\hline$\zeta_{c}$ & : Damping ratio of external work on the engine \\
\hline$\Lambda$ & : Nondimensional stiffness of deformable corrugations \\
\hline$\rho$ & : Density of working fluid $\left(\mathrm{kg} / \mathrm{m}^{3}\right)$ \\
\hline$\omega$ & : Reference frequency $(\mathrm{rad} / \mathrm{s})$ \\
\hline$\Gamma$ & : Nondimensional heating pulse \\
\hline Other Subscripts and & \\
\hline Numerical Subscript & : Thermodynamic state in the engine cycle \\
\hline Overbar & : Nondimensional term \\
\hline Overdot & : Differentiation with respect to time \\
\hline Prime & : Transient thermodynamic state in the engine cycle \\
\hline Subscript $i$ & : Nominal condition \\
\hline Subscript o & mbient condition \\
\hline
\end{tabular}




\section{References}

[1] EIA, International energy outlook 2013, Technical Report DOE/EIA0484(2013), Department of Energy, Energy Information Administration, National Energy Information Center, EI-40 U.S. Energy Information Administration, Washington, DC 20585 (July 2013).

[2] P. Capros, A. De Vita, N. Tasios, D. Papadopoulos, P. Siskos, E. Apostolaki, M. Zampara, L. Paroussos, K. Fragiadakis, N. Kouvaritakis, Eu energy, transport and ghg emissions, Tech. rep., European Comission: Directorate-General for Energy, Directorate-General for Climate Action and Directorate-General for Mobility and Transport (December 2013).

[3] J. J. V. Díaz, M. R. Wilby, A. B. R. González, The wasted energy: A metric to set up appropriate targets in our path towards fully renewable energy systems, Energy 90 (2015) 900-909.

[4] J. Heywood, Internal Combustion Engine Fundamentals, 1st Edition, McGraw-Hill, Inc, New York, 1988.

[5] S. B. Viklund, M. T. Johansson, Technologies for utilization of inn dustrial excess heat: Potentials for energy recovery and co2 emission reduction, Energy Conversion and Management 77 (0) (2014) 369 - 379. doi:http://dx.doi.org/10.1016/j.enconman.2013.09.052.

口 URL http://www.sciencedirect.com/science/article/pii/ S0196890413006134

๑ [6] M. Johansson, M. Söderström, Electricity generation from low-temperature industrial excess heat - an opportunity for the steel industry, Energy Efficiency 7 (2) (2014) 203-215. doi:10.1007/s12053-013-9218-6

URL http://dx.doi.org/10.1007/s12053-013-9218-6 
[7] S. Thapa, E. Borquist, A. Baniya, L. Weiss, Experimental and computational investigation of a mems-based boiler for waste heat recovery, Energy Conversion and Management 100 (2015) 403-413.

[8] B. Mathew, B. Jakub-Wood, E. Ogbonnaya, L. Weiss, Investigation of a mems-based capillary heat exchanger for thermal harvesting, International Journal of Heat and Mass Transfer 58 (1-2) (2013) 492-502.

[9] E. Ogbonnaya, C. Champagne, L. Weiss, Simple and low cost method for metal-based micro-capillary channels for heat exchanger use, Journal of Micromechanics and Microengineering 23 (2013) 1-10.

[10] E. Borquist, E. Ogbonnaya, S. Thapa, D. Wood, L. Weiss, Copper plated microchannel heat exchanger for mems application, Proceedings of 12th ICNMM, Chicago IL FEDSM2014 (21927) (2014) 1-6.

[11] Y. Zhao, S. Thapa, L. Weiss, Y. Lvov, Phase change insulation for energy efficiency based on wax-halloysite composites, Advanced Engineering Materials 64 (1).

[12] L. W. Weiss, Study of a mems-based free piston expander for energy sustainability, Journal of Mechanical Design 132 (9) (2010) 091002-8. doi:10.1115/1.4002083. URL http://link . aip.org/link/?JMD/132/091002/1

[13] J. Judy, Microelectromechanical systems (mems): Fabrication, design, and applications, Smart Materials and Structures 10 (2001) 1115-1134.

[14] D. C. Walther, J. Ahn, Advances and challenges in the development of power-generation systems at small scales, Progress in Energy and Combustion Science 37 (5) (2011) 583-610. 
[15] A. C. Fernandez-Pello, Micropower generation using combustion: Issues and approaches, Proceedings of the Combustion Institute 29 (1) (2002) 883 - 899. doi:DOI:10.1016/S1540-7489(02)80113-4.

[16] W. Wang, Z. Zuo, J. Liu, Miniaturization limitations of rotary internal

[17] L. Weiss, Power production from phase change in mems and micro devices, a review, International Journal of Thermal Sciences 50 (5) (2011) 639-647.

[18] M. D. Volder, D. Reynaerts, Pneumatic and hydraulic microactuators: a review, Journal of Micromechanics and Microengineering 20 (4) (2010) 043001.

[19] Y. Wang, Z. Zhou, J. Zhou, J. Liu, Z. Wang, K. Cen, Micro newcomen

[20] A. H. Epstein, Millimeter-scale, micro-electro-mechanical systems gas turbine engines, Journal of Engineering for Gas Turbines and Power 126 (2) (2004) 205-226. doi:10.1115/1.1739245

URL http://link . aip.org/link/?GTP/126/205/1

610

[21] R. T. Deam, E. Lemma, B. Mace, R. Collins, On scaling down turbines to millimeter size, Journal of Engineering for Gas Turbines and Power 130 (5) (2008) 052301. doi:10.1115/1.2938516.

[22] C. Lee, L. Frechette, A silicon microturbopump for a rankine-cycle power generation microsystem part 1: Component and system design, Micro- 
electromechanical Systems, Journal of 20 (1) (2011) $312-325$. doi: 10.1109/JMEMS. 2010.2093561.

[23] C. Lee, M. Liamini, L. Frechette, A silicon microturbopump for a rankinecycle power-generation microsystem part 2: Fabrication and characterization, Microelectromechanical Systems, Journal of 20 (1) (2011) 326 -338. doi:10.1109/JMEMS.2010.2093562

[24] M. Liamini, H. Shahriar, S. Vengallatore, L. Fré andchette, Design methodology for a rankine microturbine: Thermomechanical analysis and material selection, Microelectromechanical Systems, Journal of 20 (1) (2011) 339 -351 . doi:10.1109/JMEMS.2010.2093565.

[25] B. Tchanche, G. Lambrinos, A. Frangoudakis, G. Papadakis, Exergy analysis of micro-organic rankine power cycles for a small scale solar driven reverse osmosis desalination system, Applied Energy 15 (8) (2009) 3963-3979. doi:DOI:10.1016/j.apenergy.2009.07.011. URL http://www.sciencedirect.com/science/article/ B6V1T-4X0F6R1-1/2/c3283ada61bdf5d66d027e5bd7d9b735

[26] J. M. Zhang, E. Q. Li, S. T. Thoroddsen, A co-flow-focusing monodisperse microbubble generator, Journal of Micromechanics and Microengineering $24(3)$ (2014) 035008.

URL http://stacks .iop.org/0960-1317/24/i=3/a=035008

[27] E. Lurie, A. Kribus, Analysis of a microscale 'saturation phase-change internal carnot engine', Energy Conversion and Management 51 (6) (2010) 1202 - 1209. doi:DOI:10.1016/j.enconman.2009.12.031

口 URL http://www.sciencedirect.com/science/article/ B6V2P-4Y6T7PB-6/2/2eebda0b1022e9ef8e03ae2ef92a891d 
[30] R. Mikalsen, A. Roskilly, A review of free-piston engine history and applications, Applied Thermal Engineering 27 (14-15) (2007) 2339 - 2352. doi:DOI:10.1016/j.applthermaleng.2007.03.015.

[31] P. Achten, J. van den Oever, J. Potma, G. Vael, Horsepower with brains:

[34] H. Aichlmayr, D. Kittelson, M. Zachariah, Miniature free-piston homogenious charge compression ignition engine-compressor concept -part 2: modeling hcci combustion in small scales with detailed homogeneous gas phase chemical kinetics, Chemical Engineering Science 57 (2002) 4173-4186.

[35] Q. Wang, D. Zhang, J. Bai, Z. He, Numerical simulation of catalysis com- 
bustion inside micro free-piston engine, Energy Conversion and Management 113 (2016) 243-251.

[36] J. Bai, Q. Wang, Z. He, C. Li, J. Pan, Study on methane heci combustion process of micro free-piston power device, Applied Thermal Engineering.

[37] S. Zhang, J. Wang, Z. Guo, Novel micro free-piston swing engine and its feasibility validation, Tsinghua Science and Technology 10 (3) (2005) 381 - 386. doi:DOI : 10.1016/S1007-0214(05)70085-7.

[38] F. Formosa, L. G. Fréchette, Scaling laws for free piston stirling engine design: Benefits and challenges of miniaturization, Energy 57 (2013) 796808.

[39] B. S. Preetham, M. Anderson, C. Richards, Modeling of a resonant 675 heat engine, Journal of Applied Physics 112 (12) (2012) -. doi:http: $/ / \mathrm{dx}$. doi.org/10.1063/1.4769447 URL http://scitation.aip.org/content/aip/journal/jap/112/12/ $10.1063 / 1.4769447$

[40] B. Preetham, M. Anderson, C. Richards, Estimation of parasitic losses in a proposed mesoscale resonant engine: Experiment and model, Journal of Applied Physics 115 (5) (2014) 054904.

[41] B. Preetham, M. Anderson, C. Richards, Mathematical modeling of a fourstroke resonant engine for micro and mesoscale applications, Journal of Applied Physics 116 (21) (2014) 214904.

[42] C. Champagne, L. Weiss, Performance analysis of a miniature free piston expander for waste heat energy harvesting, Energy Conversion and Management 76 (2013) 883-892. 
[43] M. Imran, M. Usman, B.-S. Park, D.-H. Lee, Volumetric expanders for low grade heat and waste heat recovery applications, Renewable and Sustainable Energy Reviews 57 (2016) 1090-1109.

[44] J. Cho, L. Weiss, D. Bahr, C. Richards, R. Richards, Power production by a dynamic micro heat engine with an integrated thermal switch, Journal of Micromechanics and Microengineering 17 (2007) S217-S223.

[45] H. Bardaweel, R. Richards, C. Richards, M. Anderson, Cyclic operation of a mems-based resonant micro heat engine: Experiment and model, Journal of Applied Physics 107 (104901-1) (2010) 1-7. 